\title{
Does Putting a Linguist in the Loop Improve NLU Data Collection?
}

\author{
Alicia Parrish, ${ }^{1}$ William Huang, ${ }^{2 *}$ Omar Agha, ${ }^{1}$ Soo-Hwan Lee, ${ }^{1}$ Nikita Nangia, ${ }^{1}$ \\ Alex Warstadt, ${ }^{1}$ Karmanya Aggarwal, ${ }^{3}$ Emily Allaway, ${ }^{4}$ Tal Linzen, ${ }^{1}$ Samuel R. Bowman ${ }^{1}$ \\ ${ }^{1}$ New York University $\quad{ }^{2}$ Capital One $\quad{ }^{3}$ IIIT-Delhi $\quad{ }^{4}$ Columbia University
}

Correspondence: $\{a l i c i a . v \cdot p a r r i s h$, nikitanangia, linzen, bowman\}@nyu.edu

\begin{abstract}
Many crowdsourced NLP datasets contain systematic artifacts that are identified only after data collection is complete. Earlier identification of these issues should make it easier to create high-quality training and evaluation data. We attempt this by evaluating protocols in which expert linguists work 'in the loop' during data collection to identify and address these issues by adjusting task instructions and incentives. Using natural language inference as a test case, we compare three data collection protocols: (i) a baseline protocol with no linguist involvement, (ii) a linguist-in-the-loop intervention with iteratively-updated constraints on the writing task, and (iii) an extension that adds direct interaction between linguists and crowdworkers via a chatroom. We find that linguist involvement does not lead to increased accuracy on out-of-domain test sets compared to baseline, and adding a chatroom has no effect on the data. Linguist involvement does, however, lead to more challenging evaluation data and higher accuracy on some challenge sets, demonstrating the benefits of integrating expert analysis during data collection.
\end{abstract}

\section{Introduction}

Many datasets for training and evaluating natural language understanding (NLU) models consist of examples written by non-expert crowdworkers. While it is convenient and relatively inexpensive to gather large datasets from non-expert crowdworkers, the resulting datasets often suffer from systematic gaps and artifacts. Through post hoc analysis, experts have identified many such problems and found that augmenting datasets with targeted examples can mitigate these issues (Yanaka et al., 2019; Min et al., 2020). Though non-expert crowdsourcing often produces flawed data, concerns about scalability and crowdworker diversity mean there is often no viable alternative. With this

${ }^{*}$ Work done while at New York University.

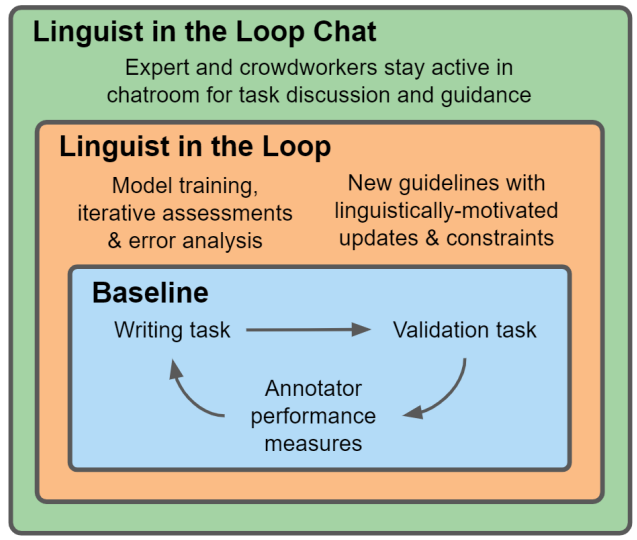

Figure 1: The three protocols compared in this study. Each crowdworker participates in only one protocol.

in mind, we investigate how to leverage expert linguistic knowledge during writing and annotation by having linguists dynamically identify artifacts and gaps in the data, then communicate with non-expert crowdworkers to instruct them towards strategies that address issues as they arise.

We focus on natural language inference (NLI; Dagan et al., 2006, i.a.), a task where the goal is to predict the label (ENTAILMENT, CONTRADICTION, NEUTRAL) that reflects the relationship of a hypothesis to a premise. For example. given the premise Jenny loves all animals, the hypothesis Jenny loves cats is an ENTAILMENT, and Jenny hates dogs, a CONTRADICTION. We choose NLI because it is among the best-studied NLU tasks, with demonstrated value (e.g., in pretraining (Clark et al., 2019)), but also multiple well-documented data quality issues that arise in crowdsourced data collection, many of which can be traced to a given heuristic. Because these heuristic-based issues are prevalent, we focus on NLI with the aim that our methodology can inform data collection for new tasks in which there are fewer known heuristics.

Previous efforts to develop more effective NLU data collection protocols have been limited in their ability to assess the efficacy of their interventions, 


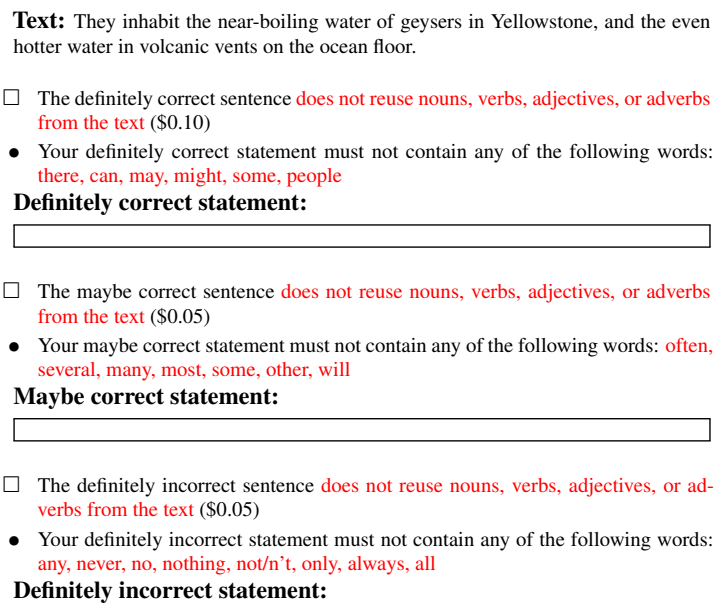

The maybe correct sentence does not reuse nouns, verbs, adjectives, or adverbs from the text $(\$ 0.05)$

- Your maybe correct statement must not contain any of the following words: often, several, many, most, some, other, will

Maybe correct statement:

The definitely incorrect sentence does not reuse nouns, verbs, adjectives, or adverbs from the text $(\$ 0.05)$

- Your definitely incorrect statement must not contain any of the following words: any, never, no, nothing, not/n't, only, always, all Definitely incorrect statement:

Figure 2: Round 5 HIT with the optional No Overlap constraint shown.

as they often lack direct comparisons between different collection methods. We directly compare three levels of expert involvement over five rounds of data collection: (i) a baseline group with no hands-on expert involvement ('Baseline'), (ii) a group that follows linguistically-motivated constraints developed by experts after each data collection round to target heuristic-based weaknesses in the data ('linguist-in-the-loop' (LitL)), and (iii) a group that extends the LitL protocol to add direct interaction with the experts, including individuallevel discussion about the task, on the chat platform Slack ('LitL Chat'). These three protocols are shown in Figure 1, and a task example with one of the constraints is shown in Figure 2.

Qualitatively, examples in each protocol appear equally free of noise (incorrect labels, typos, etc.), and lexical diversity increases in later rounds for protocols with linguist intervention. ${ }^{1}$ We find that while expert involvement (LitL and LitL Chat) does not lead to better accuracy on adversarial examples or out-of-domain datasets, it does reduce the impact of the identified artifacts and results in a more challenging final dataset, with model performances that are 5 points lower on validated data compared to Baseline. Surprisingly, we find no benefit to providing a chatroom for crowdworkers to interact directly with linguists. We recommend including expert analysis during data collection so the expert can address artifacts as they are identified.

\footnotetext{
${ }^{1}$ Appendix E contains a sample of validated examples.
}

\section{Related Work}

NLI Data Collection Methods Large-scale human-elicited datasets include the Stanford Natural Language Inference Corpus (SNLI; Bowman et al., 2015), the Multi-genre Natural Language Inference Corpus (MNLI; Williams et al., 2018), the Chinese OCNLI corpus (Hu et al., 2020), and Adversarial NLI (ANLI; Nie et al., 2020). All four datasets use non-expert crowdworkers to write hypotheses and annotate labels from pre-defined short texts, though only OCNLI and ANLI add interventions to increase data diversity. In OC$\mathrm{NLI}$, language-studies students write hypotheses in different data collection rounds with instructions for avoiding known artifacts. ANLI uses a humanand-model-in-the-loop procedure to elicit examples that are progressively more difficult for their model, resulting in a dataset with a large human-model performance gap, though identifying the cause for model failure is left up to the discretion of the worker.

Efforts to improve on sentence writing tasks for NLI have yielded mostly negative results in headto-head protocol comparisons. In an experimental comparison on different NLI crowdsourcing protocols, Vania et al. (2020) find that automatically selecting premise-hypothesis pairs for label annotation does not yield a better dataset compared to a baseline sentence writing protocol. Bowman et al. (2020) compare interventions aimed at improving NLI writing, using protocol variants that constrain the worker's task, but they see no improvements in transfer learning results compared to their baseline.

Artifacts in NLI Data Several studies have identified artifacts in NLI datasets that the models trained on them subsequently learn (often robustly). Statistical regularities in the hypothesis can allow models to assign the correct label when trained on hypothesis-only input, even though the intended task reflects the relation between the hypothesis and premise (Poliak et al., 2018; Gururangan et al., 2018 , i.a.). High lexical overlap between a premise and hypothesis is associated with a greater probability of the label being ENTAILMENT (McCoy et al., 2019; Naik et al., 2018). Additional issues in trained models suggest the presence of gaps that are harder to observe directly: Sinha et al. (2021) note the lack of syntactic understanding in NLI models as one such example, demonstrating that models often ignore syntactic information entirely. 
These diverse artifacts make NLI a good test case for protocols designed to assess issues as the data is collected.

Methods for Filling the Gaps in Datasets To collect challenging examples for NLU tasks, researchers have explored altering labeled data to create targeted or adversarial examples. Kaushik et al. (2020) have crowdworkers make minimal edits to hypotheses to align with a revised label. Gardner et al. (2020) create contrast sets for evaluation by having experts alter already-annotated examples such that the resulting label changes. Wei and Zou (2019) use simple automatic data manipulations to augment datasets for several text classification tasks, resulting in more robust models. More linguistically sophisticated manipulations have been used to augment MNLI to improve monotonicity reasoning (Yanaka et al., 2019) and to mitigate the lexical overlap heuristic (Min et al., 2020). These methods are applied after data collection is complete, so it is an open question if the gaps they identify in a final dataset would have been avoidable if addressed during data collection.

Similar to our approach, OCNLI's instructions nudge writers towards writing examples that address known artifacts. They find that encouraging the writers to follow constraints, such as avoiding negation in a CONTRADICTION label, results in a harder dataset. We expand on this work by introducing a wider range of constraints and assessing their effects throughout data collection. Our approach is also similar to Vidgen et al.'s (2021) human-generated hate-speech dataset. They introduce pivots during data collection in which they instruct crowdworkers about how to write in ways that fool their model. We expand on their method by qualitatively assessing the crowdworkers to identify issues specific to our data as it is collected.

Expert Interaction with Crowdworkers Tang et al. (2019) report that direct communication among crowdworkers leads to improved task performance on image labeling, optical character recognition, and audio transcription. This suggests that collecting higher quality data is possible when workers have real-time group interaction. Other studies have reported that interaction among crowdworkers is an effective tool for limiting some forms of bias and increasing accuracy (Drapeau et al., 2016; Schaekermann et al., 2018). In a different strategy, Roit et al. (2020) give crowdworkers detailed feedback during training, then select only a small number of those workers for the larger task, frontloading the work of the experts and relying on the selected workers to perform the task consistently.

Despite the potential benefits of real-time interaction between crowdworkers and experts, there has not yet been a direct comparison of protocols that differ based on this variable. To our knowledge, this study is both the first to test the effect of this interaction and the first head-to-head experimental assessment of human-in-the-loop data collection methods, allowing us to make conclusions about the causal effects of the different interventions compared to a baseline.

\section{Data Collection}

Task Description Our task is modeled on MNLI's data collection procedure. We present workers with a text, for which they write statements they consider definitely correct, maybe correct, and definitely incorrect. Each round of data collection creates 3,500 examples, and we collect data over five rounds. Following each round of sentence writing, crowdworkers validate 500 of the examples from their protocol. We collect four validations for each of these example and use these labels plus the original one to assign a gold label based on majority vote. Examples for which no gold label can be assigned are removed from the data. We use the validated data to evaluate our models and the unvalidated data for training. Workers with a validation rate below $70 \%$ or whose validation responses fail to match the gold label at least $70 \%$ of the time are subject to disqualification. Throughout the study, we disqualified three workers from Baseline, three from LitL, and two from LitL Chat.

Pay Structure To retain crowdworkers for all five rounds, we increase the base pay of $\$ 1 /$ HIT $^{2}$ by $\$ 0.05$ each round and pay a $\$ 20.00$ bonus after the last round. To ensure we collect sufficient examples from each worker, we award a bonus worth $10 \%$ of base pay for reaching milestones of 10, 50, and 100 HITs each round. To encourage workers to write high-quality examples, we pay a $\$ 5.00$ bonus each round to workers with over 25 HITs and at least a 95\% validation rate. We estimate that, with bonuses, a worker who completes 70 HITs per round with a high validation rate will earn $\$ 81$ in Round $1(\sim \$ 16 / \mathrm{hr})$ and $\$ 95$ in Round

\footnotetext{
2 'HIT' stands for 'Human Intelligence Task.' Each HIT is a single unit that a worker accepts via the online interface.
} 
5 ( $\$ 19 / \mathrm{hr})$. Workers in LitL and LitL Chat earn additional bonuses for completing challenge options (\$0.05-\$0.10), and workers in LitL Chat earn bonuses for participation in the chatroom $(\$ 1.50$ for any engagement, $\$ 10.00$ for active engagement).

\subsection{Crowdworker Recruitment}

We use a pre-test to recruit workers via Amazon Mechanical Turk (MTurk). The pre-test is open to workers in the United States with approval rates at or above $98 \%$ and more than 1000 HITs approved. The pre-test is a sentence-writing task where workers see a premise and write hypotheses under each of the three NLI labels. To assess if workers can follow more complicated instructions, they also write one entailed sentence that uses a conjunction and one neutral sentence that does not re-use any words from the text.

We collect responses from 155 crowdworkers, of whom 145 indicate interest in completing future, similar HITs. From those 145, we read their responses and exclude 24 for failing to adequately complete the task (many due to responses that do not follow instructions). The remaining 121 crowdworkers are retained and split between the three experimental protocols in a pseudo-random way such that (i) the three workers who asked not to participate in a chat forum are placed in the Baseline or LitL protocol, ${ }^{3}$ and (ii) groups are matched equally for workers' initial skill level based on a 4-point rating scale of their qualitative performance on the pre-test. A total of 37 crowdworkers ultimately participate in data collection in Baseline, 30 in LitL, and 32 in LitL Chat.

\subsection{Writing and Label Annotation Details}

Crowdworkers write examples and annotate labels in five rounds, with each round lasting one week and consisting of 1167 unique premises (resulting in 3501 examples). Between rounds, we conduct several planned diagnostics on our datasets to monitor the impact of our intervention and inform crowdworker feedback for the following round. All three protocols were run completely in tandem so that workers in the three protocols saw HITs become available at the same time and were sent any

\footnotetext{
${ }^{3}$ Though a potential design confound, this was necessary and had minimal effect. Requiring workers to sign up for a third party service violates Amazon's terms of service, so we allow participants to opt out. Only three participants opted out of the chat (two of whom dropped out after Round 1), and many workers placed in a non-chat protocol had indicated a willingness to participate in the chat.
}

emails or bonuses at the same time.

Writing Stage Crowdworkers construct hypotheses based on premises taken from the SLATE subset of MNLI. SLATE hosts popular culture articles from the archives of Slate Magazine. After Round 1, we exclude premises shorter than six tokens based on feedback from crowdworkers that many of the very short premises are incomplete, nonsensical, or confusing to write hypotheses for.

Diagnostic Stage After each round, we fine-tune RoBERTa (Liu et al., 2019) models using data collected up to that round. We then evaluate the models on diagnostic examples from GLUE (Wang et al., 2019) and HANS (McCoy et al., 2019). The GLUE examples target different aspects of linguistic reasoning including lexical semantics, predicateargument structure, logic, and world knowledge. HANS tests for three shallow heuristics, including lexical overlap between a premise and hypothesis. We also train and evaluate RoBERTa models using hypothesis-only input to assess artifactual cues about the label present in the hypothesis (Gururangan et al., 2018). Finally, we assess the distribution of hypothesis lengths and the pointwise mutual information (PMI) between each word in the vocabulary and label. Hypothesis length does not appear to differ by protocol or label, so it never informs our constraints.

We use these diagnostics as well as qualitative reviews of the data to devise linguistically-motivated guidelines for the following round, allowing us to adapt feedback for crowdworkers in a structured way as the data is collected. This process is conducted by five of the authors who have graduatelevel training in English syntax and semantics.

\subsection{Constraints}

Banned Words After Round 1, crowdworkers in LitL and LitL Chat are instructed not to use certain words when writing sentences for each label. We identify 5-7 banned words after each round. We use PMI to identify which words to ban under each label, as words with high label PMI are a major contributor to artifacts that allow for high performance on hypothesis-only input. We observe high PMI between existentials (e.g., there, some) and ENTAILMENT, quantificational expressions (e.g., many, often) and NEUTRAL, and negations (e.g., not, never) and CONTRADICTION. Figure 2 shows examples of the banned words during Round 5 . 


\begin{tabular}{|c|c|c|c|c|c|}
\hline \multirow[t]{2}{*}{ Constraint } & \multirow[t]{2}{*}{ Premise } & \multirow[t]{2}{*}{ Hypothesis } & \multirow[t]{2}{*}{ Label } & \multicolumn{2}{|c|}{ Attempt rate } \\
\hline & & & & LitL & Litl Chat \\
\hline $\begin{array}{l}\text { Hypernym or } \\
\text { hyponym }\end{array}$ & Does anyone know what happened to chaos? & $\begin{array}{l}\text { Whatever happened to the lack of order is } \\
\text { certainly a mystery. }\end{array}$ & $\mathrm{E}$ & 22.8 & 23.7 \\
\hline $\begin{array}{l}\text { Banned word } \\
\text { in diff. label }\end{array}$ & $\begin{array}{l}\text { Inflation is supposed to be a deadly poison, } \\
\text { not a useful medicine. }\end{array}$ & $\begin{array}{l}\text { Inflation is not supposed to be a useful } \\
\text { medicine }\end{array}$ & $\mathrm{E}$ & 43.7 & 27.7 \\
\hline $\begin{array}{l}\text { Temporal } \\
\text { reasoning }\end{array}$ & John Kasich dropped his presidential bid. & $\begin{array}{l}\text { They said that earlier, John Kasich had } \\
\text { dropped his presidential bid. }\end{array}$ & $\mathrm{E}$ & 34.1 & 10.0 \\
\hline $\begin{array}{l}\text { Synonym or } \\
\text { antonym }\end{array}$ & 2) This particular instance of it stinks. & $\begin{array}{l}\text { This instance is perceived to be a good } \\
\text { thing. }\end{array}$ & $\mathrm{C}$ & 39.5 & 24.5 \\
\hline All overlap & $\begin{array}{l}\text { News argues that most of America's } 93 \text { mil- } \\
\text { lion volunteers aren't doing much good. }\end{array}$ & $\begin{array}{l}\text { News argues that volunteers aren't doing } \\
\text { much good. }\end{array}$ & $\mathrm{E}$ & 21.8 & 30.4 \\
\hline $\begin{array}{l}\text { Register } \\
\text { change }\end{array}$ & $\begin{array}{l}\text { First, the horsemen brought out a teaser } \\
\text { horse. }\end{array}$ & $\begin{array}{l}\text { Teaser horses are commonly thought to be } \\
\text { both entertaining and tragic. }\end{array}$ & $\mathrm{N}$ & 25.3 & 15.0 \\
\hline No overlap & and she doesn't floss while driving. & The woman has an automated car. & $\mathrm{N}$ & 29.2 & 22.3 \\
\hline $\begin{array}{l}\text { Relative } \\
\text { clause }\end{array}$ & $\begin{array}{l}\text { Sun Ra's spaceships did not come, as it were, } \\
\text { out of nowhere. }\end{array}$ & $\begin{array}{l}\text { The spaceships that belong to Sun Ra came } \\
\text { out of nowhere }\end{array}$ & $\mathrm{C}$ & 35.0 & 24.3 \\
\hline $\begin{array}{l}\text { Reverse argu- } \\
\text { ment order }\end{array}$ & After an inquiry regarding Bob Dole's ... & $\begin{array}{l}\text { It is illegal for Bob Dole to receive in- } \\
\text { quiries. }\end{array}$ & $\mathrm{N}$ & 36.7 & 29.4 \\
\hline $\begin{array}{l}\text { Grammar } \\
\text { change }\end{array}$ & $\begin{array}{l}\text { The Bush campaign has a sweet monopoly } \\
\text { on that. }\end{array}$ & $\begin{array}{l}\text { The Obama campaign had a sweet } \\
\text { monopoly on that. }\end{array}$ & $\mathrm{C}$ & 22.6 & 13.4 \\
\hline Sub-part & $\begin{array}{l}\text { He was crying like his mother had just wal- } \\
\text { loped him. }\end{array}$ & $\begin{array}{l}\text { He cried a lot, as though he were walloped } \\
\text { on his behind. }\end{array}$ & $\mathrm{E}$ & 23.2 & 19.1 \\
\hline $\begin{array}{l}\text { Background } \\
\text { knowledge }\end{array}$ & $\begin{array}{l}\text { In both Britain and America, the term cov- } \\
\text { ers nearly everybody. }\end{array}$ & $\begin{array}{l}\text { The term generally applied to countries in } \\
\text { two opposite sides of the world. }\end{array}$ & $\mathrm{E}$ & 32.9 & 15.9 \\
\hline
\end{tabular}

Table 1: Sentence pairs displaying each challenge option. Where applicable, relevant contrasts are bolded. Examples are randomly drawn from data that passed validation on the constraint with the restriction that both sentences be fewer than 80 characters ( $\sim 32 \%$ of the data). The last column shows the percentage of the challenges attempted.

Challenge Options We use constraints, framed as challenge options to the worker, to target heuristics that we identify in the data during the diagnostic state. By explicitly telling workers to avoid these heuristics, we aim to lower their contribution to any artifacts in the final dataset. We determine constraints through qualitative assessment of the data, taking into consideration syntactic diversity, lexical choice, and semantic or world-knowledgebased reasoning patterns. For example, after noticing that the majority of hypotheses relied only on the stated information from the premise in Round 1 , we encouraged workers in Round 2 to focus on "background knowledge" (last example in Table 1) that they know to be true, but isn't explicitly stated, such as the knowledge that Britain and America are countries on opposite sides of the world. The 12 challenge options are defined in Appendix A, with examples of each in Table 1. After Round 1, each HIT in LitL and LitL Chat lists one constraint. Assignment of the constraints was completely random and not based on features of individual premises, but each constraint was presented as a possible option an approximately equal number of times across HITs. This task is optional for the workers, as some constraints are incompatible with some examples.

\subsection{Protocols}

Baseline Protocol Our Baseline protocol follows the task description in $\S 3$ and does not include any direct expert involvement. Crowdworker performance is only measured via validation.

Linguist-in-the-Loop (LitL) Protocol LitL extends the Baseline protocol with constraints (described in §3.3). As the constraints make the task more difficult, we award bonuses of $\$ 0.05-\$ 0.10$ per example to workers who indicate that they attempted the challenge option. The bonus amount is determined by the linguists' assessment of the difficulty; for example, the No Overlap constraint is more difficult to apply in entailment examples than neutral, so a No Overlap entailment example has a higher bonus. During validation, crowdworkers also label whether each example adheres to the challenge constraint (the interface is shown in Appendix F). For any worker whose validation rate on the challenges is below $50 \%$, we contact them to explain the source of their errors.

LitL Chat Protocol We provide direct communication with expert linguists on Slack. We encourage workers to ask task-specific questions for anything they find challenging or confusing, and we encourage active discussion to help workers 
better understand the task. Most questions seek to clarify if a certain strategy 'counts' as adhering to a constraint. Feedback given via email in the LitL protocol is instead given via direct message in Slack, unless the worker initiates contact over email, as was sometimes the case for logistical issues. Additionally, at the beginning of Rounds 3-5, we identify creative examples written in a previous round and post them to Slack for inspiration, with a brief comment. These interactions on Slack are the only difference between the LitL and LitL Chat protocols.

\subsection{Crowdworker Performance}

Inter-Annotator Agreement Baseline shows the highest inter-annotator agreement on NLI labels with a Krippendorf's $\alpha$ of 0.709 , while LitL and LitL Chat have 0.655 and 0.640 , respectively. All three meet the standard threshold for "substantial agreement." We calculate Krippendorf's $\alpha$ because it is both appropriate for nominal data and robust to missing values (Zapf et al., 2016), i.e., cases where not every worker rates every item. Validation rates for the NLI labels are $93.7 \%$ for Baseline, $89.76 \%$ for LitL, and $91.36 \%$ for LitL Chat. LitL and LitL Chat may have slightly lower validation rates than Baseline because the constraints lead to challenging examples, making the validator's task more difficult.

Frequency of Constraint Attempts The attempt rate of bonus challenges differs between constraints (Table 1). Overall, more abstract categories (e.g., background knowledge) are attempted less often than more concrete constraints. There are also differences by protocol, as LitL has a higher attempt rate than LitL Chat, possibly because workers in LitL Chat are more selective in identifying appropriate examples to apply the constraints to. Supporting this potential explanation, we find that LitL Chat had higher constraint validation rates than LitL in Rounds 4 and 5, indicating that workers in LitL Chat adhered to the constraints more accurately after practice.

Use of Slack The total number of active workers on Slack fell from 23 in Round 1 to just 16 by Round $4 .{ }^{4}$ The total number of messages sent also fell with each round, going from about 215 posts and replies in Round 1 to 162 in Round 4. It may

\footnotetext{
${ }^{4}$ Round 5 was even lower, but spanned the US Thanksgiving holiday, which likely artificially lowered participation.
}

be that workers rely on the chat less as they become more familiar with the task. Though only about half of the workers in LitL Chat participated in the Slack channel, the workers who were active on Slack also completed a high number of HITs; if the chatroom has a reliable effect on the data created by workers using it, then we expect this effect to still be measurable. Further, though we heavily incentivized use of the Slack channel, the fact that many workers still chose not to use it reveals that this low participation rate may be a typical outcome on micro-task platforms such as MTurk.

\section{Modeling Experiments}

For each round and protocol, we collect 3.5k examples and use the 500 validated examples as validation data and the remaining $3 \mathrm{k}$ for training. ${ }^{5}$ We then fine-tune a RoBERTa $\mathrm{Lg}_{\text {(Large) model }}$ on all the data accumulated up to that round. For example, the Round 2 model is trained on examples from Rounds 1 and 2 with training and validation sizes of $6 \mathrm{k}$ and $1 \mathrm{k}$, respectively. We also fine-tune a RoBERTa ${ }_{\text {Lg }}$ model previously trained on MNLI (RoBERTa $\left.a_{L g+M N L I}\right)$, though results are consistently similar to RoBERTa $\mathrm{Lg}_{\mathrm{Lg}}$ (details in Appendix B). After each round, we evaluate our models on the diagnostics described in $\$ 3.2$.

Estimating Confidence Intervals We estimate average accuracy and confidence intervals by finetuning 10 additional models with a sample of $90 \%$ of the collected training data. We use the best hyperparameters for each protocol and round from our hyperparameter search described below. In sampling the data, we first sort the data by crowdworker and successively remove $10 \%$ of examples, allowing us to study variation among workers while controlling for training set size. This design choice also helps account for the potential issue of overestimating performance due to having the same writers for the training and test sets (Geva et al., 2019), as the successive removal of $10 \%$ of the training data simulates the removal of all or most of a single worker's writing from the train set, but not the test set, similarly in all protocols.

Implementation To fine-tune our models, we perform a grid search over learning rate $\in\{5 e-$ $6,1 e-5,2 e-5,3 e-5\}$ and batch size $\in\{16,32\}$ and use the hyperparameters yielding the best in-

\footnotetext{
${ }^{5}$ Data and code are available at https://github.com/AliciaParrish/ling_in_loop
} 


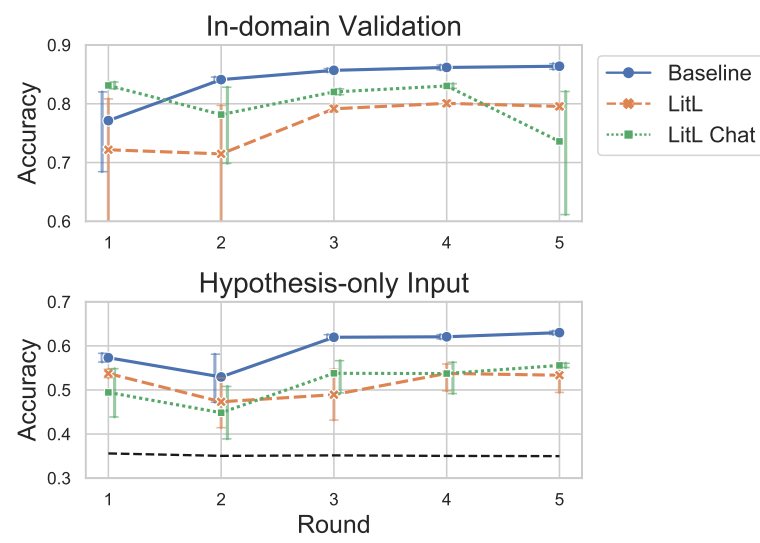

Figure 3: Performance of RoBERTa ${ }_{L g}$ fine-tuned on data collected through different protocols on validation data from the same protocol, configured normally (top) or using only the hypothesis (bottom). For each round, we include training and validation data accumulated up to Round $n$. The dashed black line marks the average majority class baseline across protocols. Error bars represent bootstrapped confidence intervals.

domain validation accuracy. We train for 20 epochs, since each round of data collection yields $3 \mathrm{k}$ training examples, and longer training has been shown to help smaller training sets (Zhang et al., 2020). Our code is based on jiant (Phang et al., 2020), which uses PyTorch (Paszke et al., 2019) and Transformers (Wolf et al., 2020).

\subsection{Results}

Evaluation Set Difficulty We test whether data collected with expert intervention leads to a more challenging test set by comparing in-domain performance for each protocol for RoBERTa $\mathrm{Lg}_{\mathrm{L}}$, using the validated evaluation data accumulated up to that round (Figure 3). This allows us to study the characteristics of an iteratively collected corpus using cumulative rounds in each protocol. We see that LitL and LitL Chat performance falls below Baseline after the introduction of linguistically-informed constraints in Round 2. Figure 5 shows a similar trend - performance from RoBERTa Lg $_{\text {fine-tuned only on }}$ $M N L I$ on the validation sets decreases or remains lower for LitL and LitL Chat, while performance on Baseline increases as more data is collected. As we evaluate on validated examples, it is unlikely that this lower performance is due to noise in the data. Rather, these findings indicate we are able to create more challenging evaluation data using the LitL and LitL Chat interventions, with LitL slightly outperforming LitL Chat.
Hypothesis-Only Performance We test whether the data collected with linguist intervention leads to a reduction in artifacts that contribute to high performance on hypothesis-only input. We compare accuracy for each protocol for RoBERTa $_{\mathrm{Lg}}$ trained on hypothesis-only input, where lower accuracy suggests fewer artifacts in the data (Figure 3). Both LitL and LitL Chat show lower accuracy than Baseline, and this gap widens in later rounds. To assess whether this widening from Round 1 to 5 is statistically reliable, we conduct a two-way ANOVA of round by protocol, which yields an interaction $(p=0.049)$, indicating that while hypothesis-only performance increases for all protocols with more training examples, this increase in artifacts is significantly reduced in LitL and LitL Chat compared to Baseline. The lower rate of artifacts in LitL and LitL Chat may be due to the lower average word-label PMI, which increases over rounds for Baseline while consistently falling in both LitL and LitL Chat. ${ }^{6}$ However, for all protocols, accuracies are still above chance performance, leaving room to further reduce these artifacts.

Diagnostic Sets We evaluate whether fine-tuning on data collected with linguist involvement leads to a model that has higher performance on challenge test sets. Figure 4 shows model performance on the GLUE diagnostic set and HANS non-entailment examples. A two-way ANOVA of round by protocol does not reveal any significant interactions or main effects for GLUE. For HANS, we see higher accuracy from LitL and LitL Chat for Lexical Overlap and Subsequence examples in Rounds 4 and 5 after introducing No and All Overlap constraints, though the interaction is only significant with RoBERTa $\operatorname{Lg}_{\mathrm{L}+\mathrm{MNLI}}\left(p_{\text {corr }}=0.0147\right.$ and $p_{\text {corr }}$ $=0.0119$ for Lexical Overlap and Subsequence, respectively, after applying Bonferroni correction to correct for 7 comparisons against the same null hypothesis (Cabin and Mitchell, 2000)), despite the visually larger accuracy increases in RoBERTa $\mathrm{L}_{\mathrm{Lg}}$. This is likely due to greater variance in the data, indicating that there may be strong effects of individual workers on lexical overlap and subsequence biases. Performance on HANS entailment examples are in line with McCoy et al. (2019) with median accuracies of $90 \%$ or higher (Appendix D).

To investigate if lexical overlap rates differ by

\footnotetext{
${ }^{6} \mathrm{~A}$ two-way ANOVA again reveals a significant interaction of protocol by round ( $p=0.022$ ) on word-label PMI values.
} 

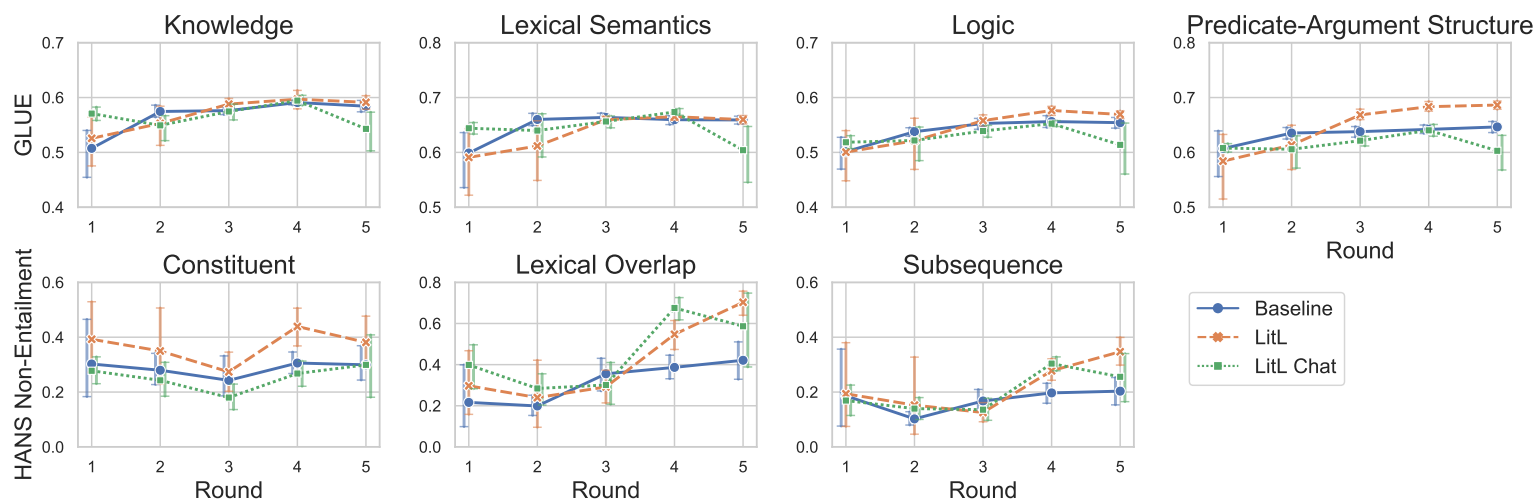

......... LitL Chat

Figure 4: Performance of RoBERTa $\mathrm{Lg}$ fine-tuned on data collected through different protocols on the GLUE diagnostic set (top) and HANS non-entailment examples (bottom). Error bars represent bootstrapped confidence intervals.

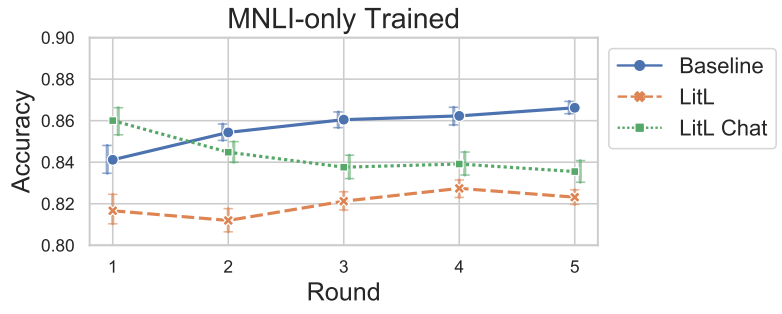

Figure 5: Average performance of RoBERTa ${ }_{\mathrm{Lg}}$ finetuned on MNLI (not our data) over 10 random restarts on validated examples accumulated up to Round $n$. Error bars represent bootstrapped confidence intervals.

protocol, we assess classification accuracy for a linear model trained only on the example's overlap rate, defined as the proportion of words in the hypothesis that are also in the premise. We observe that any artifactual cues introduced from overlap rate are strongest in the Baseline protocol, which performs 9.52 points above majority class guessing, while LitL and LitL Chat perform 8.06 and 6.88 points above majority class guessing, respectively.

Held-Out Evaluation Sets After the final round of data collection, we test whether models finetuned on data collected with linguist involvement show better out-of-domain performance by evaluating models trained on our data on MNLImismatched $^{7}$ (Williams et al., 2018) and ANLI (Nie et al., 2020). Evaluating on held-out sets allows us to test if our interventions lead to increased model accuracy on datasets generated through different protocols or from different sources while

\footnotetext{
${ }^{7}$ The MNLI corpus includes two evaluation sets, MNLImatched and MNLI-mismatched, with examples sourced from different genres. We evaluate on MNLI-mismatched, as we source our premise sentences from an MNLI-matched genre.
}
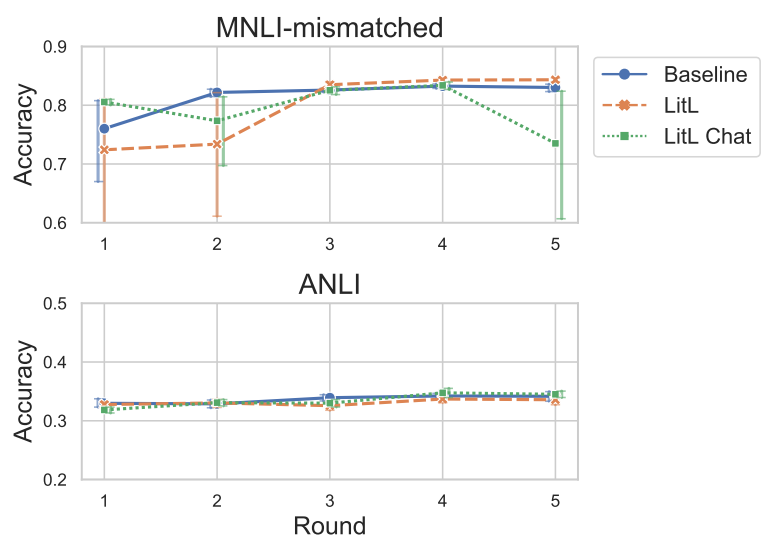

Figure 6: Performance of RoBERTa $\mathrm{Lg}_{\mathrm{Lg}}$ fine-tuned on data collected through different protocols on MNLImismatched (top) and ANLI (bottom). Error bars represent bootstrapped confidence intervals.

ensuring that we do not overly tune our feedback to these benchmarks. Figure 6 shows that there is little difference in ANLI and MNLI-mismatched performance between models trained with data from different protocols. The high variability in Round 5 accuracy for LitL Chat may be due to artifacts from just one or two crowdworkers, highlighting the importance of estimating individual workers' effects on a final dataset. We perform a more granular analysis on ANLI examples using the tags from Williams et al. (2020) and again find no clear effect of protocol (details in Appendix C). Even though our interventions reduce some artifacts in the hypothesis and improve model performance on HANS non-entailment examples, we have no evidence that these benefits transfer to out-of-domain examples or examples from adversarial protocols. 


\section{Considerations in Choosing a Protocol}

In broad terms, we observe a benefit from dynamically updating instructions and incentives to address artifacts identified during data collection. This procedure increased the average cost per example by $4.1 \%$ over an average base cost of $\$ 0.367$. We offered $\$ 0.05$ to $\$ 0.10$ per example, but given the somewhat low rate at which crowdworkers chose to attempt the challenges (28.6\% and $21.2 \%$ for LitL and LitL Chat, respectively), we find it likely that increasing the amount offered per example would have increased participation, potentially also increasing the benefits observed in model performance. In an exit survey, over $50 \%$ of workers in LitL and LitL Chat indicated that they would have completed more optional challenges if the pay had been higher. We recommend that future work using challenge options offer bonuses worth at least $15 \%$ of the base pay.

Cost of Linguist Involvement The iterative analyses and updates to the guidelines in LitL and LitL Chat protocols took 10-12 hours of expert time per week, compared to one hour per week to monitor task completion in Baseline. The use of Slack nearly doubled the expert time needed, adding an additional 8-10 hours each week for LitL Chat over LitL, even after taking into account the slight reduction in time spent replying to email questions that shifted to Slack. If we value linguist time at $\$ 40 / \mathrm{hr}$, this raises the final price per example to $\$ 0.378$ in Baseline, with LitL $31.2 \%$ higher, and LitL Chat $58.5 \%$ higher.

Qualitative Considerations Though many crowdworkers in LitL Chat expressed that they enjoyed the extra communication, crowdworkers from LitL and LitL Chat rated the task as 'more enjoyable' than typical MTurk tasks at nearly identical rates $(85.2 \%$ and $87.5 \%$ respectively, compared to $67.7 \%$ in Baseline). Workers' ratings of the difficulty of writing and validation tasks were also nearly identical among the three protocols. We therefore find that, for typical data collection on MTurk, the addition of a chat platform to facilitate worker-expert interaction is ineffective at improving data quality.

\section{Conclusion}

Having experts review and analyze incoming crowdsourced data during data collection allows those experts to identify new areas of weakness at each round and update guidelines and constraints while there is still time for those interventions to lessen the impact of artifacts in the data. Though we do not observe any increases in out-of-domain accuracy, linguist involvement leads to more challenging evaluation data and higher accuracy on some challenge sets in HANS. One-on-one interactions between experts and crowdworkers, though reported in some studies as being beneficial for more challenging tasks, has no measurable effect in our study. Future work could extend the expertinvolved protocol to identify additional interventions that would lead to datasets with better generalizability.

\section{Acknowledgments}

This project has benefited from financial support to SB by Eric and Wendy Schmidt (made by recommendation of the Schmidt Futures program), Samsung Research (under the project Improving Deep Learning using Latent Structure), Apple, and Intuit, and from in-kind support by the NYU HighPerformance Computing Center and by NVIDIA Corporation (with the donation of a Titan V GPU). This material is based upon work supported by the National Science Foundation under Grant Nos. 1922658 and 2046556. Any opinions, findings, and conclusions or recommendations expressed in this material are those of the author(s) and do not necessarily reflect the views of the National Science Foundation.

\section{Ethical Considerations}

Typical MTurk tasks pay well below a living wage for the US, with median earnings at only about $\$ 2 / \mathrm{hr}$ (Hara et al., 2018). Though we target a fair wage of $\$ 15 / \mathrm{hr}$, MTurk as a whole is not designed to ensure fair pay for its workers. We detail our estimates of worker pay to make it clear that we ensured a fair rate, but we recognize that any work using this platform has the potential to encourage more 'typical' low-paying tasks. Additionally, we did not control for crowdworker demographics nor did we explicitly give workers instructions about avoiding social biases in their writing. There is therefore no reason to expect that training a system on data collected via the protocol we advocate for here will result in a model that is more fair. 


\section{References}

Samuel R. Bowman, Gabor Angeli, Christopher Potts, and Christopher D. Manning. 2015. A large annotated corpus for learning natural language inference. In Proceedings of the 2015 Conference on Empirical Methods in Natural Language Processing (EMNLP), pages 632-642.

Samuel R. Bowman, Jennimaria Palomaki, Livio Baldini Soares, and Emily Pitler. 2020. Collecting entailment data for pretraining: New protocols and negative results. In Proceedings of the 2020 Conference on Empirical Methods in Natural Language Processing (EMNLP), pages 8203-8214.

Robert J Cabin and Randall J Mitchell. 2000. To bonferroni or not to bonferroni: when and how are the questions. Bulletin of the ecological society of America, 81(3):246-248.

Christopher Clark, Kenton Lee, Ming-Wei Chang, Tom Kwiatkowski, Michael Collins, and Kristina Toutanova. 2019. Boolq: Exploring the surprising difficulty of natural yes/no questions. In Proceedings of the 2019 Conference of the North American Chapter of the Association for Computational Linguistics: Human Language Technologies, Volume 1 (Long and Short Papers), pages 2924-2936.

Ido Dagan, Oren Glickman, and Bernardo Magnini. 2006. The PASCAL recognising textual entailment challenge. In Machine Learning Challenges Workshop, pages 177-190. Springer.

Ryan Drapeau, Lydia Chilton, Jonathan Bragg, and Daniel Weld. 2016. Microtalk: Using argumentation to improve crowdsourcing accuracy. In Proceedings of the AAAI Conference on Human Computation and Crowdsourcing, volume 4.

Matt Gardner, Yoav Artzi, Victoria Basmova, Jonathan Berant, Ben Bogin, Sihao Chen, Pradeep Dasigi, Dheeru Dua, Yanai Elazar, Ananth Gottumukkala, Nitish Gupta, Hanna Hajishirzi, Gabriel Ilharco, Daniel Khashabi, Kevin Lin, Jiangming Liu, Nelson F. Liu, Phoebe Mulcaire, Qiang Ning, Sameer Singh, Noah A. Smith, Sanjay Subramanian, Reut Tsarfaty, Eric Wallace, Ally Zhang, and Ben Zhou. 2020. Evaluating models' local decision boundaries via contrast sets. In Proceedings of the 2020 Conference on Empirical Methods in Natural Language Processing: Findings, pages 1307-1323.

Mor Geva, Yoav Goldberg, and Jonathan Berant. 2019. Are we modeling the task or the annotator? an investigation of annotator bias in natural language understanding datasets. In Proceedings of the 2019 Conference on Empirical Methods in Natural Language Processing and the 9th International Joint Conference on Natural Language Processing (EMNLPIJCNLP), pages 1161-1166.

Suchin Gururangan, Swabha Swayamdipta, Omer Levy, Roy Schwartz, Samuel R. Bowman, and Noah A. Smith. 2018. Annotation artifacts in natural language inference data. In Proceedings of the 2018 Conference of the North American Chapter of the Association for Computational Linguistics: Human Language Technologies, NAACL-HLT, New Orleans, Louisiana, USA, June 1-6, 2018, Volume 2 (Short Papers), pages 107-112. Association for Computational Linguistics.

Kotaro Hara, Abigail Adams, Kristy Milland, Saiph Savage, Chris Callison-Burch, and Jeffrey P Bigham. 2018. A data-driven analysis of workers' earnings on Amazon Mechanical Turk. In Proceedings of the 2018 CHI conference on human factors in computing systems, pages 1-14.

Hai Hu, Kyle Richardson, Liang Xu, Lu Li, Sandra Kübler, and Lawrence S Moss. 2020. OCNLI: Original chinese natural language inference. In Proceedings of the 2020 Conference on Empirical Methods in Natural Language Processing: Findings, pages 3512-3526.

Divyansh Kaushik, Eduard Hovy, and Zachary Lipton. 2020. Learning the difference that makes a difference with counterfactually-augmented data. In 8th International Conference on Learning Representations, ICLR 2020.

Yinhan Liu, Myle Ott, Naman Goyal, Jingfei Du, Mandar Joshi, Danqi Chen, Omer Levy, Mike Lewis, Luke Zettlemoyer, and Veselin Stoyanov. 2019. RoBERTa: A robustly optimized BERT pretraining approach. arXiv preprint arXiv:1907.11692.

Tom McCoy, Ellie Pavlick, and Tal Linzen. 2019. Right for the wrong reasons: Diagnosing syntactic heuristics in natural language inference. In Proceedings of the 57th Annual Meeting of the Association for Computational Linguistics, pages 3428-3448, Florence, Italy. Association for Computational Linguistics.

Junghyun Min, R. Thomas McCoy, Dipanjan Das, Emily Pitler, and Tal Linzen. 2020. Syntactic data augmentation increases robustness to inference heuristics. In Proceedings of the 58th Annual Meeting of the Association for Computational Linguistics, pages 2339-2352, Online. Association for Computational Linguistics.

Aakanksha Naik, Abhilasha Ravichander, Norman M. Sadeh, Carolyn Penstein Rosé, and Graham Neubig. 2018. Stress test evaluation for natural language inference. In Proceedings of the 27th International Conference on Computational Linguistics, COLING 2018, Santa Fe, New Mexico, USA, August 20-26, 2018, pages 2340-2353. Association for Computational Linguistics.

Yixin Nie, Adina Williams, Emily Dinan, Mohit Bansal, Jason Weston, and Douwe Kiela. 2020. Adversarial NLI: A new benchmark for natural language understanding. In Proceedings of the 58th Annual Meeting of the Association for Computational Linguistics, ACL 2020, Online, July 5-10, 2020, 
pages 4885-4901. Association for Computational Linguistics.

Adam Paszke, Sam Gross, Francisco Massa, Adam Lerer, James Bradbury, Gregory Chanan, Trevor Killeen, Zeming Lin, Natalia Gimelshein, Luca Antiga, Alban Desmaison, Andreas Kopf, Edward Yang, Zachary DeVito, Martin Raison, Alykhan Tejani, Sasank Chilamkurthy, Benoit Steiner, Lu Fang, Junjie Bai, and Soumith Chintala. 2019. PyTorch: An Imperative Style, High-Performance Deep Learning Library. In H. Wallach, H. Larochelle, A. Beygelzimer, F. d' Alché-Buc, E. Fox, and R. Garnett, editors, Advances in Neural Information Processing Systems 32, pages 8024-8035. Curran Associates, Inc.

Jason Phang, Phil Yeres, Jesse Swanson, Haokun Liu, Ian F. Tenney, Phu Mon Htut, Clara Vania, Alex Wang, and Samuel R. Bowman. 2020. jiant 2.0: A software toolkit for research on general-purpose text understanding models. htt $\mathrm{p}$ : //jiant.info/.

Adam Poliak, Jason Naradowsky, Aparajita Haldar, Rachel Rudinger, and Benjamin Van Durme. 2018. Hypothesis only baselines in natural language inference. In Proceedings of the Seventh Joint Conference on Lexical and Computational Semantics, pages 180-191, New Orleans, Louisiana. Association for Computational Linguistics.

Paul Roit, Ayal Klein, Daniela Stepanov, Jonathan Mamou, Julian Michael, Gabriel Stanovsky, Luke Zettlemoyer, and Ido Dagan. 2020. Controlled crowdsourcing for high-quality QA-SRL annotation. In Proceedings of the 58th Annual Meeting of the Association for Computational Linguistics, pages 7008-7013.

Mike Schaekermann, Joslin Goh, Kate Larson, and Edith Law. 2018. Resolvable vs. irresolvable disagreement: A study on worker deliberation in crowd work. Proceedings of the ACM on HumanComputer Interaction, 2(CSCW):1-19.

Koustuv Sinha, Prasanna Parthasarathi, Joelle Pineau, and Adina Williams. 2021. UnNatural Language Inference. In Proceedings of the 59th Annual Meeting of the Association for Computational Linguistics and the 11th International Joint Conference on Natural Language Processing (Volume 1: Long Papers), pages 7329-7346, Online. Association for Computational Linguistics.

Wei Tang, Ming Yin, and Chien-Ju Ho. 2019. Leveraging peer communication to enhance crowdsourcing. In The World Wide Web Conference, WWW 2019, San Francisco, CA, USA, May 13-17, 2019, pages 1794-1805. ACM.

Clara Vania, Ruijie Chen, and Samuel R. Bowman. 2020. Asking Crowdworkers to Write Entailment Examples: The Best of Bad Options. In Proceedings of the 1st Conference of the Asia-Pacific Chapter of the Association for Computational Linguistics and the 10th International Joint Conference on Natural Language Processing, Online. Association for Computational Linguistics.

Bertie Vidgen, Tristan Thrush, Zeerak Waseem, and Douwe Kiela. 2021. Learning from the worst: Dynamically generated datasets to improve online hate detection. In Proceedings of the 59th Annual Meeting of the Association for Computational Linguistics and the 11th International Joint Conference on Natural Language Processing (Volume 1: Long Papers), pages 1667-1682, Online. Association for Computational Linguistics.

Alex Wang, Amanpreet Singh, Julian Michael, Felix Hill, Omer Levy, and Samuel R. Bowman. 2019. GLUE: A multi-task benchmark and analysis platform for natural language understanding. In 7 th International Conference on Learning Representations, ICLR 2019, New Orleans, LA, USA, May 6-9, 2019.

Jason Wei and Kai Zou. 2019. EDA: Easy data augmentation techniques for boosting performance on text classification tasks. In Proceedings of the 2019 Conference on Empirical Methods in Natural Language Processing and the 9th International Joint Conference on Natural Language Processing (EMNLP-IJCNLP), pages 6382-6388, Hong Kong, China. Association for Computational Linguistics.

Adina Williams, Nikita Nangia, and Samuel Bowman. 2018. A broad-coverage challenge corpus for sentence understanding through inference. In Proceedings of the 2018 Conference of the North American Chapter of the Association for Computational Linguistics: Human Language Technologies, Volume 1 (Long Papers), pages 1112-1122. Association for Computational Linguistics.

Adina Williams, Tristan Thrush, and Douwe Kiela. 2020. ANLIzing the adversarial natural language inference dataset. arXiv preprint arXiv:2010.12729.

Thomas Wolf, Lysandre Debut, Victor Sanh, Julien Chaumond, Clement Delangue, Anthony Moi, Pierric Cistac, Tim Rault, Remi Louf, Morgan Funtowicz, Joe Davison, Sam Shleifer, Patrick von Platen, Clara Ma, Yacine Jernite, Julien Plu, Canwen Xu, Teven Le Scao, Sylvain Gugger, Mariama Drame, Quentin Lhoest, and Alexander Rush. 2020. Transformers: State-of-the-art natural language processing. In Proceedings of the 2020 Conference on Empirical Methods in Natural Language Processing: System Demonstrations, pages 38-45, Online. Association for Computational Linguistics.

Hitomi Yanaka, Koji Mineshima, Daisuke Bekki, Kentaro Inui, Satoshi Sekine, Lasha Abzianidze, and Johan Bos. 2019. HELP: A dataset for identifying shortcomings of neural models in monotonicity reasoning. In Proceedings of the Eighth Joint Conference on Lexical and Computational Semantics (*SEM 2019), pages 250-255, Minneapolis, Minnesota. Association for Computational Linguistics. 
Antonia Zapf, Stefanie Castell, Lars Morawietz, and André Karch. 2016. Measuring inter-rater reliability for nominal data-which coefficients and confidence intervals are appropriate? BMC medical research methodology, 16(1):1-10.

Tianyi Zhang, Felix Wu, Arzoo Katiyar, Kilian Q. Weinberger, and Yoav Artzi. 2020. Revisiting few-sample BERT fine-tuning. arXiv preprint arXiv:2006.05987.

\section{A List of Challenge Options}

For each challenge option, we present workers with the name of the constraint and a brief explanation of what it means. The brief explanation is followed by a longer 2-3 sentence explanation that includes a concrete example, such as showing what a relative clause is or giving an example of a hypernym/hyponym pair.

\section{Lexical Options}

- Temporal reasoning (Round 2): The hypothesis should reference two separate time points.

- Restricted word in different label (Round 2): The hypothesis should contain a word that is banned for a different label.

- Hypernym or hyponym (Rounds $2 \& 3$ ): The hypothesis should contain a hypernym or hyponym (a more or less specific word or phrase) of a word in the premise.

- Synonym or antonym (Rounds $2 \& 3$ ): The hypothesis should contain a synonym or antonym of a word in the premise.

- No overlap (Rounds 4 \& 5): The hypothesis should use none of the content words appearing in the premise. Content words are nouns, verbs, adjectives, and adverbs.

- All overlap (Rounds 4 \& 5): The hypothesis should only use content words that appear in the premise. Introducing new function words is allowed, as is changing grammatical features of the content words.

\section{Syntactic Options}

- Relative clause (Round 2): The hypothesis should contain a relative clause. A relative clause is a noun that is described by a phrase that begins with words like who or that.
- Reverse argument order (Rounds $2 \& 3$ ): The hypothesis should contain a pair of noun phrases from the premise in reverse order.

- Grammar change (Round 4): The hypothesis should change a grammatical element of the premise, such as tense, number, or gender on a pronoun.

\section{World Knowledge Options}

- Background knowledge (Rounds 2 \& 4): The hypothesis should target background facts or general knowledge that workers can infer from the premise.

- Sub-part (Round 3): The hypothesis should refer to something that is a part of an entity in the premise. For example, sub-parts of a bus include its steering wheel and engine.

- Register change (Round 5): The hypothesis should differ from the original text in its level of formality.

\section{B MNLI-Pretrained RoBERTa Results}

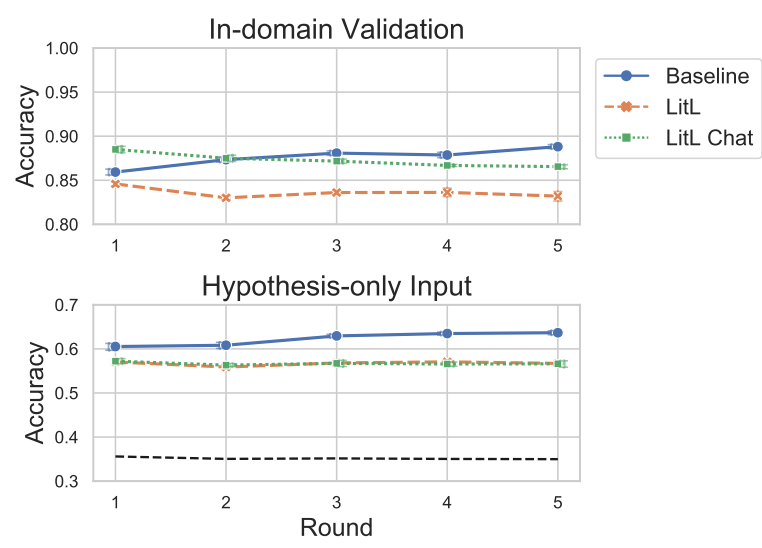

Figure 7: Performance of RoBERTa ${ }_{\mathrm{Lg}+\mathrm{MNLI}}$ finetuned on data collected through different protocols on in-domain validation data trained with either the full example (top) or hypothesis-only (bottom) input. Higher hypothesis-only accuracy indicates a greater effect of artifacts. For each round, we include training and validation data accumulated up to Round $n$. Dashed black line marks average majority class baseline across protocols. Error bars represent bootstrapped confidence intervals.

We fine-tune a RoBERTa ${ }_{L g}$ model previously trained on MNLI (RoBERTa $a_{L}+$ MNLI $)$ on the same 


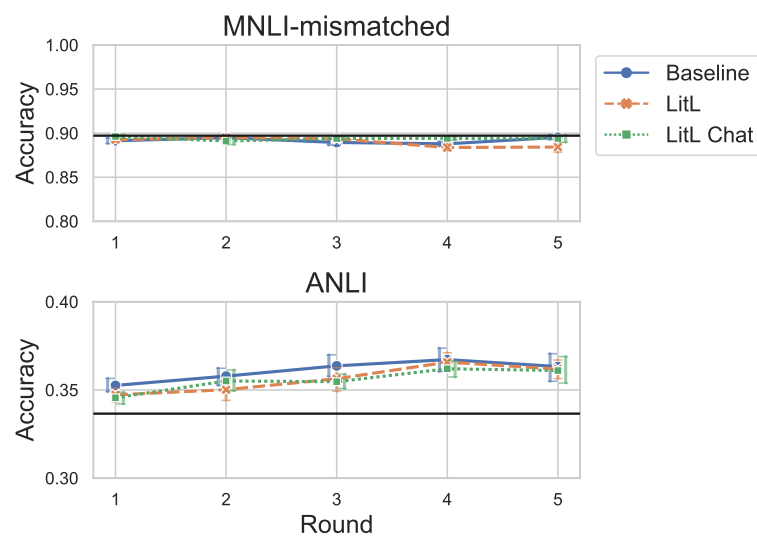

Figure 8: Performance of RoBERTa $\mathrm{L}_{\mathrm{L}+\mathrm{MNLI}}$ finetuned on data collected through different protocols on MNLI-mismatched (top) and ANLI (bottom). The black line for MNLI-mismatched and ANLI indicates performance of RoBERTa ${ }_{L g}$ fine-tuned on MNLI alone. Error bars represent bootstrapped confidence intervals.

sets of training data used for the RoBERTa ${ }_{L g}$ analyses. We find similar trends to those from finetuning $\mathrm{RoBERTa}_{\mathrm{Lg}}$ and report them in the analogous plots here.

Figure 7 shows the performance of RoBERTa $_{L g+M N L I}$ fine-tuned using either the full example or hypothesis-only input. For both types of input, we see a performance gap between Baseline and our intervention protocols. We perform a two-way ANOVA of round by protocol to see if this performance gap significantly changes between rounds 1 and 5 and find a significant interaction $(p<0.001$ for both full example and hypothesis-only input). For the full example input, this indicates that our interventions create more challenging evaluation data. For hypothesis-only performance, Baseline performance increases while LitL and LitL Chat remain relatively unchanged, indicating that our interventions mitigate stronger hypothesis-only artifacts in NLI datasets as new data is collected.

Figure 8 shows the performance of RoBERTa $_{L g+M N L I}$ fine-tuned on each protocol on MNLI-mismatched and ANLI. We find no significant difference among protocols for either held-out set.

Figure 9 shows the performance of RoBERTa $_{L g+M N L I}$ fine-tuned on data from each protocol on the GLUE diagnostic set and HANS non-entailment examples. For the GLUE diagnostic set, we do not find any significant difference among protocols. For the HANS examples, we perform a two-way ANOVA of round by protocol and find significant interaction terms for all HANS categories $\left(p_{\text {corr }}=0.0126\right.$, 0.0147, 0.0119 for Constituent, Lexical Overlap, and Subsequence, respectively, after applying Bonferroni correction for 7 tests against the same null hypothesis). For Lexical Overlap and Subsequence, these findings indicate our interventions lead to higher accuracy compared to Baseline. For the Constituent examples, the data from each protocol is especially noisy, with larger error bars and more dramatic changes in performance between rounds; it is unclear whether this is due to our protocol or the types of examples that the Constituent subset of HANS uses.

\section{ANLI Performance by Reasoning Type}

We test whether any of the reasoning tags in ANLI (Williams et al., 2020) reveal an area where data collection with linguist involvement leads to improved model performance. Figure 10 shows the performances of RoBERTa $\mathrm{Lg}_{\mathrm{g}}$ and RoBERTaLg+MNLI fine-tuned on our data and tested on ANLI by reasoning tag. Similar to our findings in Figures 6 and 8, we do not find any increases in accuracy from our interventions for any reasoning tags.

\section{HANS Entailment Peformance}

On the entailment subset of HANS, models typically achieve accuracies near $100 \%$ McCoy et al. (2019). This is because the three heuristics in HANS target instances that lead to a greater likelihood of the model choosing ENTAILMENT compared to NEUTRAL or CONTRADICTION, and thus the non-entailment portion of HANS is the challenge set. Figure 11 shows the performance of RoBERTa $_{L g}$ and RoBERTa $a_{L g}+$ MNLI fine-tuned on our data and tested on HANS entailment examples. For RoBERTa $a_{L}$, variability in performance reduces in later rounds as the training set size grows with $3 \mathrm{k}$ examples per round, though median performances for all rounds are still $90 \%$ or higher. For RoBERTa $a_{L g}+\mathrm{MNLI}$, accuracies are near $100 \%$, consistent with McCoy et al.'s findings.

\section{E Examples of Collected Data}

In order to show a representative sample of the validated data, we randomly sample premises from Round 5 data for which annotations exist in all three labels for each protocol (roughly $45 \%$ of that 

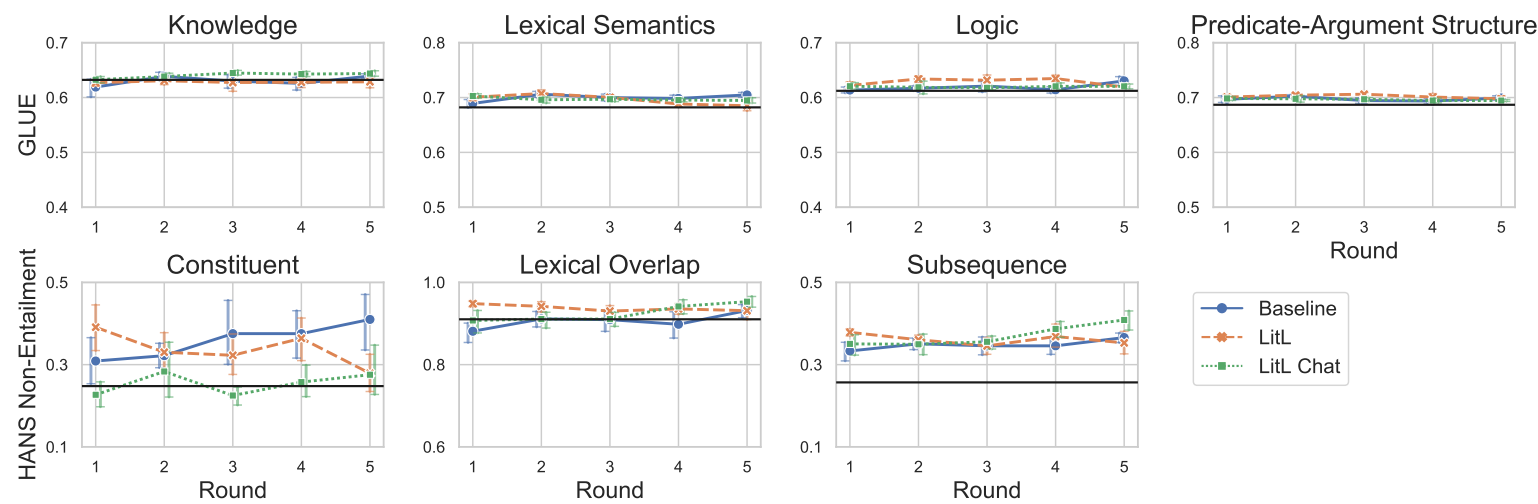

$\rightarrow$ Baseline

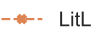

......... LitL Chat

Figure 9: Performance of RoBERTa $\mathrm{Lg}_{\mathrm{L}+\mathrm{MNLI}}$ fine-tuned on data collected through different protocols on the GLUE diagnostic set (top) and HANS non-entailment examples (bottom). The black line indicates performance of RoBERTa $_{\mathrm{Lg}}$ fine-tuned on MNLI alone. Error bars represent bootstrapped confidence intervals.

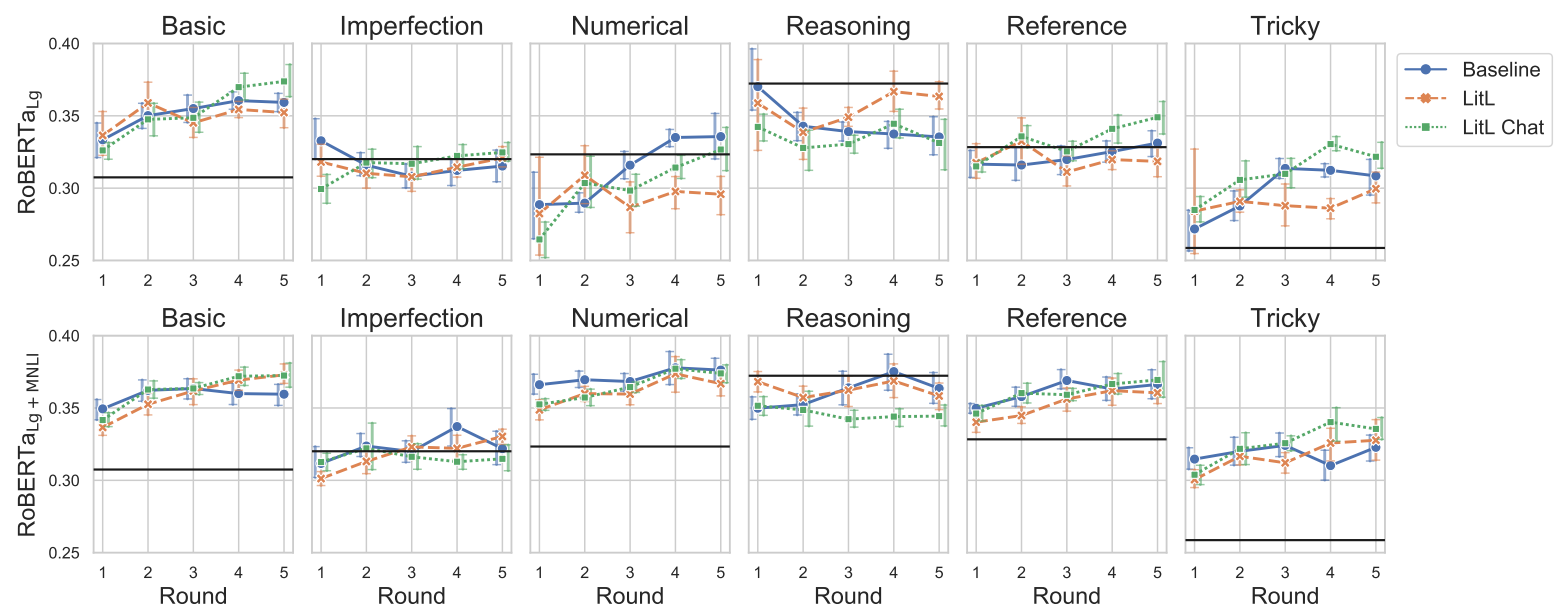

Figure 10: Performance of RoBERTa ${ }_{L g}$ (top) and RoBERTa $a_{L g}+$ MNLI (bottom) fine-tuned on data collected through different protocols on ANLI by reasoning tag from Williams et al. (2020). The black line indicates performance of a RoBERTa $_{L g}$ trained on MNLI alone. Error bars represent bootstrapped confidence intervals.

round's validated data). Five such examples are presented in Table 2. Example complexity varies widely from example to example, and it is not always the case that the example in Baseline is the simplest one. For premise 4, for example, the Baseline crowdworker has written very complex examples that require abstract reasoning about the knowledge that Harris has. For this same premise, the LitL Chat crowdworker has also created a tricky set of examples, in this case ones that do not re-use any words from the original premise.

In premise 3, we see an example where the LitL Chat crowdworker uses the idiom seen better days for the entailment example, in place of just using a different lexical item for tough as the crowdworkers in the other two protocols do. Use of idioms was suggested to workers in LitL and LitL Chat as one way to write more creative examples. In premise 5, we see that the LitL crowdworker has written a challenging contradiction example, one which requires knowledge that if help is needed on a project, that means it must not be complete.

\section{F Validation Task Interfaces}

Figure 12 provides an example of the validation interface used by the Baseline protocol throughout the study, and by LitL and LitL Chat in rounds 1 before constraints were introduced. Each HIT contained six such examples.

Figure 13 provides an example of the validation interface used by LitL and LitL Chat in rounds 2 through 5. Each HIT contained six such examples. The only difference between this and Figure 12 is that, in these HITs, workers are also prompted to validate whether the constraint was followed for that example. 

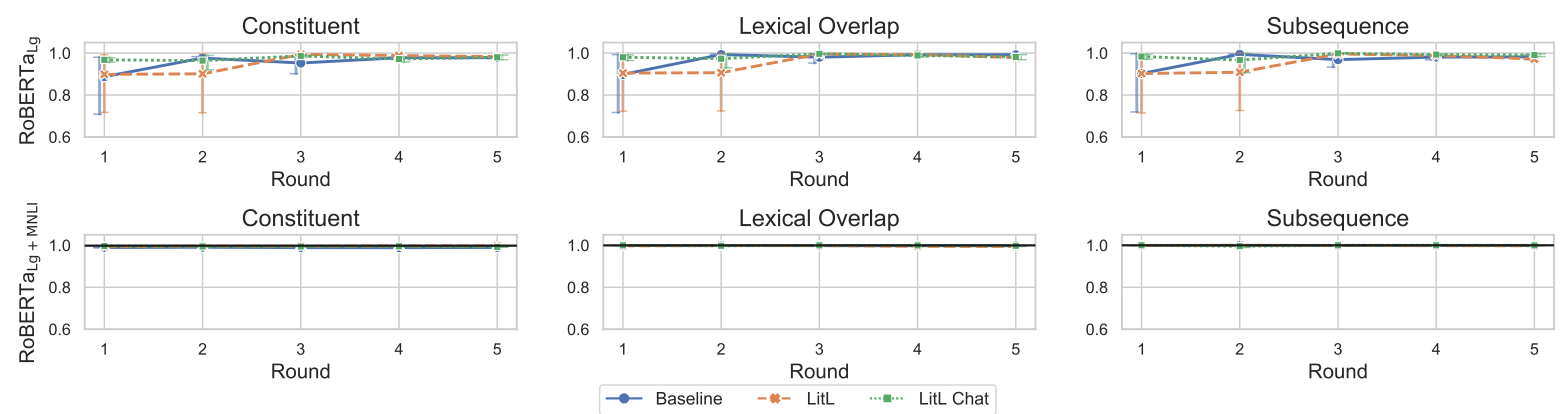

Figure 11: Performance of RoBERTa $\operatorname{Lg}_{\text {(top) and }}$ RoBERTa $\mathrm{Lg}_{\mathrm{L}+\mathrm{MNLI}}$ (bottom) fine-tuned on data collected through different protocols on HANS entailment examples. The black line indicates performance of a RoBERTa $\mathrm{Lg}_{\text {g }}$ trained on MNLI alone. Error bars represent bootstrapped confidence intervals.

Text: Trump, who said he would decide by March whether to run for president, would likely spend 100 millionto 200 million of his own money on a campaign.

Statement: Trump was considering a presidential campaign.

The statement about the text is:

Definitely correct Maybe correct Definitely incorrect

Figure 12: Example question from a validation HIT used for Baseline throughout the study, and for LitL and LitL Chat in round 1 before the introduction of challenge options.

Text: The story also made the front page of the New York Times and the Financial Times of London, which said that more than 10,000 members of a mystic cult called Fa Lun Gong caused acute embarrassment to security forces by virtually surrounding the compound where China's leaders work.

Statement: Security forces were embarrassed by a cult in China.

The statement about the text is:

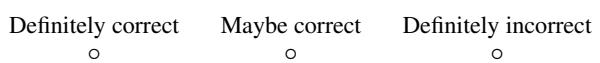

For the statement above, does the following constraint apply the statement relies on something that is not explicitly stated, but is part of common knowledge

$\begin{array}{cc}\text { Yes } & \text { No } \\ \circ & \circ\end{array}$

Figure 13: Example question from a validation HIT that includes validation of the challenge options. This task was used with LitL and LitL Chat after round 1, once we had introduced challenge options into the task. 


\begin{tabular}{|c|c|c|c|c|c|}
\hline & \multirow[t]{2}{*}{ Premise } & \multirow[t]{2}{*}{ Label } & \multicolumn{3}{|c|}{ Hypothesis } \\
\hline & & & Baseline & LitL & LitL Chat \\
\hline \multirow[t]{4}{*}{1} & \multirow{4}{*}{$\begin{array}{l}\text { (The Ramseys } \\
\text { buried their } \\
\text { daughter in Atlanta, } \\
\text { then vacationed in } \\
\text { Sea Island, Ga.) } \\
\text { This absence, some } \\
\text { speculate, gave the } \\
\text { Ramseys time to } \\
\text { work out a story to } \\
\text { explain their } \\
\text { innocence. }\end{array}$} & E & $\begin{array}{l}\text { Some people were skeptical } \\
\text { of the Ramseys' reasons for } \\
\text { going on vacation. }\end{array}$ & $\begin{array}{l}\text { The Ramseys came up with } \\
\text { a story to tell the media they } \\
\text { didn't do it. }\end{array}$ & $\begin{array}{l}\text { Some speculate that the } \\
\text { Ramseys worked out a story } \\
\text { while on vacation. }\end{array}$ \\
\hline & & $\mathrm{N}$ & $\begin{array}{l}\text { The Ramsey's held a pri- } \\
\text { vate funeral service for their } \\
\text { daughter }\end{array}$ & $\begin{array}{l}\text { The Ramseys had nothing } \\
\text { to hide. }\end{array}$ & $\begin{array}{l}\text { The Ramseys worked in At- } \\
\text { lanta. }\end{array}$ \\
\hline & & $\mathrm{C}$ & $\begin{array}{l}\text { daughter. } \\
\text { The Ramsey's daughter } \\
\text { joined them on their trip to } \\
\text { Sea Island. }\end{array}$ & $\begin{array}{l}\text { The Ramseys went into } \\
\text { mourning after burying } \\
\text { their daughter. }\end{array}$ & $\begin{array}{l}\text { The Ramseys buried their } \\
\text { daughter in Sea Island, Ga. }\end{array}$ \\
\hline & & & & & \\
\hline \multirow[t]{3}{*}{2} & \multirow{3}{*}{$\begin{array}{l}\text { Mr. Clinton rewards } \\
\text { Mr. Knight for his } \\
\text { fund raising, Mr. } \\
\text { Gore lays the } \\
\text { groundwork for his } \\
\text { anticipated } \\
\text { presidential bid four } \\
\text { years from now, and } \\
\text { the companies, by } \\
\text { hiring Mr. Knight, } \\
\text { get the } \\
\text { administration's ear. }\end{array}$} & E & $\begin{array}{l}\text { Al Gore planned to run for } \\
\text { president. }\end{array}$ & $\begin{array}{l}\text { Mr. Gore lays the ground- } \\
\text { work for his anticipated } \\
\text { presidential bid four years } \\
\text { from now. }\end{array}$ & $\begin{array}{l}\text { By hiring Mr. Knight, com- } \\
\text { panies were listened to by } \\
\text { the administration. }\end{array}$ \\
\hline & & $\mathrm{N}$ & $\begin{array}{l}\text { Companies were hopeful } \\
\text { they could get Clinton to } \\
\text { further reduce corporate tax } \\
\text { rates. }\end{array}$ & $\begin{array}{l}\text { Mr. Knight get the adminis- } \\
\text { tration's ear for companies } \\
\text { that contribute to his fund } \\
\text { raising. }\end{array}$ & $\begin{array}{l}\text { The administration had } \\
\text { been ignoring the compa- } \\
\text { nies up to this point. }\end{array}$ \\
\hline & & $\mathrm{C}$ & $\begin{array}{l}\text { Bill Clinton punished Mr. } \\
\text { Knight because of his fund } \\
\text { raising efforts. }\end{array}$ & $\begin{array}{l}\text { Mr. Clinton admonishes } \\
\text { Mr. Knight for his fund rais- } \\
\text { ing. }\end{array}$ & $\begin{array}{l}\text { Companies were ignored } \\
\text { by the adminstration be- } \\
\text { cause of the hiring of Mr. } \\
\text { Knight. }\end{array}$ \\
\hline \multirow[t]{3}{*}{3} & \multirow{3}{*}{$\begin{array}{l}\text { And these are tough } \\
\text { times for reviewers } \\
\text { in general. }\end{array}$} & E & $\begin{array}{l}\text { Reviewers are going } \\
\text { through difficult times. }\end{array}$ & $\begin{array}{l}\text { Reviewers are having a } \\
\text { challenging time. }\end{array}$ & $\begin{array}{l}\text { Reviewers have seen better } \\
\text { days. }\end{array}$ \\
\hline & & $\mathrm{N}$ & $\begin{array}{l}\text { The recession is to blame } \\
\text { for these tough times. }\end{array}$ & $\begin{array}{l}\text { Times will only get tougher } \\
\text { for reviewers. }\end{array}$ & $\begin{array}{l}\text { Reviewers are still able to } \\
\text { get by. }\end{array}$ \\
\hline & & $\mathrm{C}$ & $\begin{array}{l}\text { This is a great time to be a } \\
\text { reviewer. }\end{array}$ & $\begin{array}{l}\text { Reviewers have rarely had } \\
\text { it so easy. }\end{array}$ & $\begin{array}{l}\text { This have to be the best } \\
\text { time to get into the review } \\
\text { game. }\end{array}$ \\
\hline \multirow[t]{3}{*}{4} & \multirow{3}{*}{$\begin{array}{l}\text { To some critics, the } \\
\text { mystery isn't, as } \\
\text { Harris suggests, } \\
\text { how women } \\
\text { throughout history } \\
\text { have exploited their } \\
\text { sexual power over } \\
\text { men, but how pimps } \\
\text { like him have come } \\
\text { away with the profit. }\end{array}$} & E & $\begin{array}{l}\text { The author argues that } \\
\text { some critics are incapable } \\
\text { of understanding the role } \\
\text { pimps have played in the ex- } \\
\text { ploitation of women. }\end{array}$ & $\begin{array}{l}\text { pimps like him have prof- } \\
\text { ited. }\end{array}$ & $\begin{array}{l}\text { An unsolved question in- } \\
\text { volves the money making } \\
\text { of a hustler. }\end{array}$ \\
\hline & & $\mathrm{N}$ & $\begin{array}{l}\text { If women are going to at- } \\
\text { tempt to exploit their sex- } \\
\text { ual power over men, then } \\
\text { it is only natural for pimps } \\
\text { to emerge to oversee sexual } \\
\text { transactions. }\end{array}$ & $\begin{array}{l}\text { Pimps have exploited } \\
\text { women who have more } \\
\text { power than they think. }\end{array}$ & $\begin{array}{l}\text { Reviewers are mainly con- } \\
\text { cerned with hustlers. }\end{array}$ \\
\hline & & $\mathrm{C}$ & $\begin{array}{l}\text { Harris does not understand } \\
\text { the means by which women } \\
\text { have using sexual power in } \\
\text { order to exploit men. }\end{array}$ & $\begin{array}{lll}\text { Pimps control every } \\
\text { woman. }\end{array}$ & $\begin{array}{l}\text { An unsolved question in- } \\
\text { volves the money wasting } \\
\text { of a hustler. }\end{array}$ \\
\hline \multirow[t]{3}{*}{5} & \multirow{3}{*}{$\begin{array}{l}\text { We need your help } \\
\text { with another new } \\
\text { feature that starts } \\
\text { next week. }\end{array}$} & E & $\begin{array}{l}\text { Next week, a new feature } \\
\text { will be introduced. }\end{array}$ & $\begin{array}{l}\text { There have been other new } \\
\text { features. }\end{array}$ & $\begin{array}{l}\text { We are starting a new fea- } \\
\text { ture next week. }\end{array}$ \\
\hline & & $\mathrm{N}$ & $\begin{array}{l}\text { This new feature focuses on } \\
\text { cloud technology. }\end{array}$ & $\begin{array}{l}\text { Help has been needed with } \\
\text { previous features. }\end{array}$ & $\begin{array}{l}\text { We are starting a new fea- } \\
\text { ture next week that uses } \\
\text { maps. }\end{array}$ \\
\hline & & $\mathrm{C}$ & $\begin{array}{l}\text { The new feature will start } \\
\text { six months from now. }\end{array}$ & $\begin{array}{l}\text { The project is complete and } \\
\text { currently unsupported. }\end{array}$ & $\begin{array}{l}\text { We have more help than we } \\
\text { need for the new feature } \\
\text { next week. }\end{array}$ \\
\hline
\end{tabular}

Table 2: Randomly selected examples from validation data showing typical writing from each protocol. 